\title{
Research on control system of automatic welding robot for space intersection
}

\author{
Kekuan Wang ${ }^{1, a}$, Mingzhu Liu, ${ }^{1, b}$, Bin Long ${ }^{1, c}$ and Ruibin Duan ${ }^{1, d}$ \\ ${ }^{1}$ CNPC Research Institute of Engineering Technology Tianjin, P. R. China \\ awangkekuan@cnpc.com.cn, ${ }^{b}$ liumz@cnpc.com.cn, ${ }^{c}$ longb@cnpc.com.cn, ${ }^{d}$ duanrb@cnpc.com.cn
}

Keywords: spatial intersection line, multi axis control, saddle shaped trajectory, control system. Abstract. Based on the typical T joint full position automatic welding, the mathematical model of the saddle shaped trajectory of the intersection line is established, and the drive system of the multi dimension and multi degree of freedom is designed. The control system uses the distributed control mode, based on kinematics analysis to perform system, using multi axis controller as the control core, using VC++ language as the language, and realizes the complex motion of space intersecting line welding execution system based on multi axis.

\section{Introduction}

The development of offshore oil and gas resources, will promote large-scale platform and its main structures of jacket construction scale, large-scale construction technology requirements to improve the steel strength level, increase the diameter and wall thickness is increased, resulting in the increase of space intersection line welding difficulty and the work, with the welding quality put forward higher requirements. At the height of the development of automatic welding technology, automatic welding jacket space intersection lines has become the rapid growth of offshore oil development needs, and the multi axis control technology is the key technology of space intersection line welding.

Based on the research of the space trajectory intersection line motion space, the mathematical model was established, based on the design of jacket space intersecting line of multi axis automatic welding control system, to achieve cross line space efficient automatic welding.

\section{Motion system analysis}

Motion space trajectory analysis. In the steel structure, the T-type joint-line is a line of intersection line. The intersection line is a space curve, and it is still in a different angle in the actual node, as shown in Figure 1. The automatic welding, the welding torch moving trajectory is relatively complex. It involves the control of multi-dimensional and multi degree of freedom, and also involves the welding process of different welding positions.

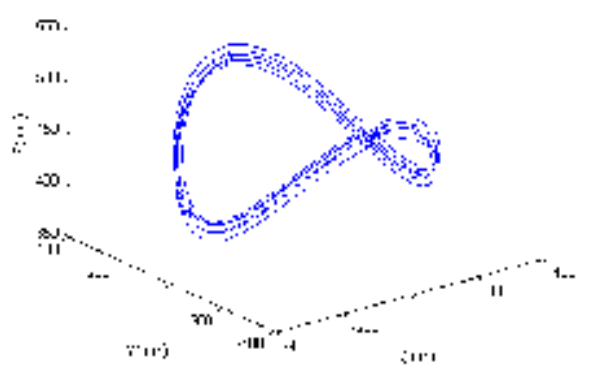

Fig.1 Saddle shaped space trajectory

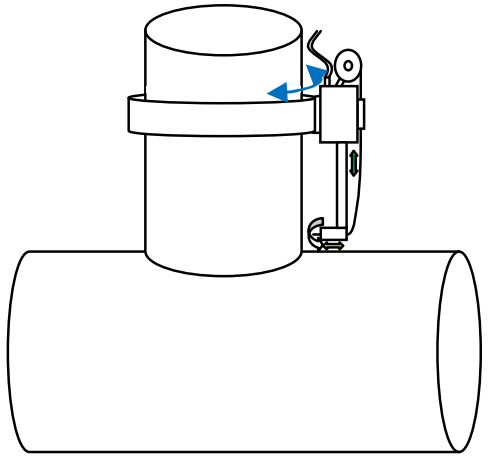

Fig.2 Motion system analysis diagram

Because the pipeline by the offshore platform construction in the thick wall, in the process of welding by multi pass welding, so the welding system to complete the action, the gun head along the saddle curve movement is multi degree of freedom space movement. To achieve more freedom of movement on the need for multi axis drive, and use the body to achieve the various decomposition of 
the action. As shown in Figure 2, the saddle shaped trajectory decomposition of the action included: the circumferential direction of the vertical tube along the axis of motion, along the vertical axis of the cantilever rod of the reciprocating motion, the stem elongation control movement, the gun head and the weld angle control.

The establishment of mathematical model of trajectory. The research object of the intersection line of space intersection is the two column orthogonal, and the intersecting line is a typical saddle shape curve. First of all, the intersecting line of two orthogonal cylinder is divided into a number of discrete points between each two points by linear interpolation, in order to ensure weld curve fitting with the actual weld match, take the calculation between two points connected to the maximum height of the weld seam, the division is greater than the set value to the curve, and then calculate the programming location and other features.

For the two orthogonal cylinder, the model of the workpiece is set up as shown in Figure 3, where $\mathrm{XYZ}$ is the reference coordinate system, and the characteristic coordinate system of the intersecting line is shown in Figure 3.

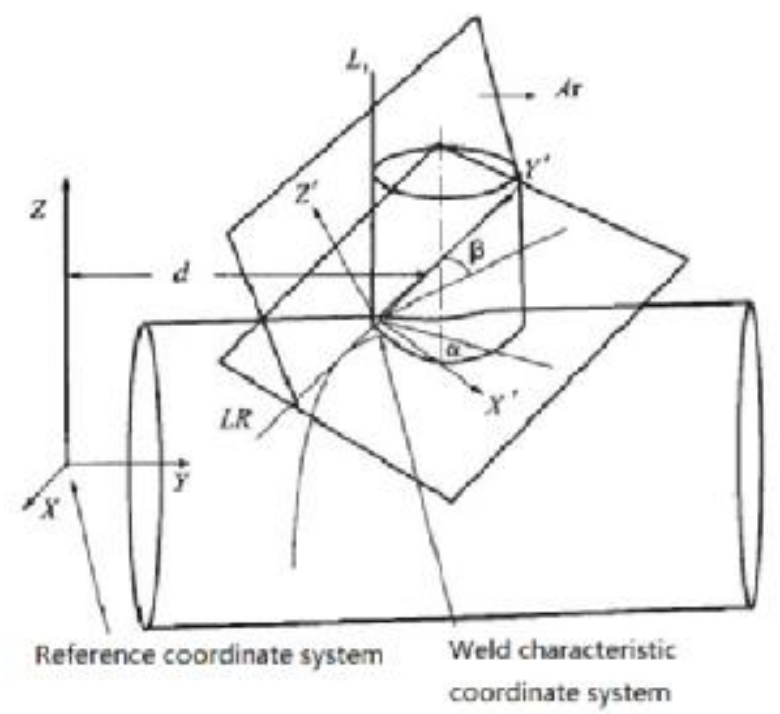

Fig.3 workpiece model

The equation for a vertical cylinder is:

$$
(\mathrm{Y}-\mathrm{d})^{2}+\mathrm{X}^{2}=\mathrm{r}^{2}
$$

The equation for a horizontal cylinder is:

$$
Z^{2}+X^{2}=R^{2}
$$

The intersection of two cylinders is:

$$
\left\{\begin{array}{c}
(Y-d)^{2}+X^{2}=r^{2}(Z>0) \\
Z^{2}+X^{2}=R^{2}
\end{array}\right.
$$

Here, the definition of perpendicular to the $\mathrm{X}$ axis and passing through the point (X0, $\mathrm{Y} 0, \mathrm{Z} 0$ ) plane to plane Ar method; definition method of plane Ar and vertical cylindrical intersection in point (X0, Y0, Z0) of the tangent Lr; definition method of plane Ar and the intersection point of the horizontal cylinder in (X0, Y0, the tangent is LR Z0).

It can be an arbitrary point on the line, $(\mathrm{X} 0, \mathrm{Y} 0, \mathrm{Z} 0)$ of the tangent equation is:

$$
\frac{X-X_{0}}{\left|\begin{array}{cc}
2\left(Y_{0}-d\right) & 0 \\
0 & 2 Z_{0}
\end{array}\right|}=\frac{Y-Y_{0}}{\left|\begin{array}{cc}
0 & 2 X_{0} \\
2 Z_{0} & 2 X_{0}
\end{array}\right|}=\frac{Z-Z_{0}}{\left|\begin{array}{cc}
2 X_{0} & 2\left(Y_{0}-d\right) \\
2 X_{0} & 0
\end{array}\right|}
$$


Intersection method of plane and vertical cylinder Ar

$$
\left\{\begin{array}{c}
(\mathrm{Y}-\mathrm{d})^{2}+\mathrm{X}^{2}=\mathrm{r}^{2} \\
-\mathrm{Z}_{0}(\mathrm{Y}-\mathrm{d})\left(\mathrm{X}-\mathrm{X}_{0}\right)+\mathrm{X}_{0} \mathrm{Z}_{0}\left(\mathrm{Y}-\mathrm{Y}_{0}\right)+\mathrm{X}_{0}\left(\mathrm{Y}_{0}-\mathrm{d}\right)\left(\mathrm{Z}-\mathrm{Z}_{0}\right)=0
\end{array}\right.
$$

The direction of the tangent $\mathrm{Lr}$ projection vector

$$
\mathrm{X}_{0}\left(\mathrm{Y}_{0}-\mathrm{d}\right)^{2},-\mathrm{X}_{0}^{2}\left(\mathrm{Y}_{0}-\mathrm{d}\right), \mathrm{X}_{0}^{2} \mathrm{Z}_{0}+\left(\mathrm{Y}_{0}-\mathrm{d}\right)^{2} \mathrm{Z}_{0}
$$

Direction projection vector of tangent $\mathrm{Lr}$

$$
\left\{\begin{array}{c}
\mathrm{Z}^{2}+\mathrm{X}^{2}=\mathrm{R}^{2} \\
-\mathrm{Z}_{0}(\mathrm{Y}-\mathrm{d})\left(\mathrm{X}-\mathrm{X}_{0}\right)+\mathrm{X}_{0} \mathrm{Z}_{0}\left(\mathrm{Y}-\mathrm{Y}_{0}\right)+\mathrm{X}_{0}\left(\mathrm{Y}_{0}-\mathrm{d}\right)\left(\mathrm{Z}-\mathrm{Z}_{0}\right)=0
\end{array}\right.
$$

The direction of the tangent LR projection vector

$$
\left[\mathrm{X}_{0}\left(\mathrm{Y}_{0}-\mathrm{d}\right)^{2} \mathrm{~A}-\mathrm{Z}_{0}^{2} \mathrm{X}_{0} \mathrm{~B}\right] \mathrm{l}-\left[\mathrm{Z}_{0}^{2}\left(\mathrm{Y}_{0}-\mathrm{d}\right) \mathrm{B}+\mathrm{X}_{0}^{2}\left(\mathrm{Y}_{0}-\mathrm{d}\right)(\mathrm{A}+\mathrm{B})\right] \mathrm{m}+\left[\mathrm{Z}_{0} \mathrm{X}_{0}^{2} \mathrm{~A}+\mathrm{Z}_{0}^{2}\left(\mathrm{Y}_{0}-\mathrm{d}\right) \mathrm{A}+\mathrm{Z}_{0}^{2} \mathrm{X}_{0} \mathrm{~B}\right] \mathrm{n}=0
$$

Where,

$$
\begin{aligned}
& A=\sqrt{X_{0}^{2} Z_{0}^{2}+\left(Y_{0}-d\right)^{2}\left(Z_{0}^{2}+X_{0}^{2}\right)^{2}+X_{0}^{4} Z_{0}^{2}}, \\
& B=\sqrt{X_{0}^{2}\left(Y_{0}-d\right)^{4}+X_{0}^{4}\left(Y_{0}-d\right)^{2}+\left[Z_{0} X_{0}^{2}+Z_{0}\left(Y_{0}-d\right)^{2}\right]^{2}}
\end{aligned}
$$

So the linear Lz equation is:

$$
\left\{\begin{array}{c}
-\mathrm{Z}_{0}\left(\mathrm{Y}_{0}-\mathrm{d}\right) 1+\mathrm{X}_{0} \mathrm{Z}_{0} \mathrm{~m}+\mathrm{X}_{0}\left(\mathrm{Y}_{0}-\mathrm{d}\right) \mathrm{n}=0 \\
{\left[\mathrm{X}_{0}\left(\mathrm{Y}_{0}-\mathrm{d}\right)^{2} \mathrm{~A}-\mathrm{Z}_{0}^{2} \mathrm{X}_{0} \mathrm{~B}\right] \mathrm{l}-\left[\mathrm{Z}_{0}^{2}\left(\mathrm{Y}_{0}-\mathrm{d}\right) \mathrm{B}+\mathrm{X}_{0}^{2}\left(\mathrm{Y}_{0}-\mathrm{d}\right)(\mathrm{A}+\mathrm{B})\right] \mathrm{m}} \\
+\left[\mathrm{Z}_{0} \mathrm{X}_{0}^{2} \mathrm{~A}+\mathrm{Z}_{0}^{2}\left(\mathrm{Y}_{0}-\mathrm{d}\right) \mathrm{A}+\mathrm{Z}_{0}^{2} \mathrm{X}_{0} \mathrm{~B}\right] \mathrm{n}=0
\end{array}\right.
$$

In summary the homogeneous transformation matrix between the space curve at any point of the weld coordinate and reference coordinate system is:

$$
R=\left|\begin{array}{cccc}
r(1,1) & r(2,1) & r(3,1) & X_{0} \\
r(1,2) & r(2,2) & r(3,2) & Y_{0} \\
r(1,3) & r(2,3) & r(3,3) & Z_{0} \\
0 & 0 & 0 & 1
\end{array}\right|
$$

Thus, the mathematical model of the welding trajectory of the intersection line is established, according to the mathematical model, the appropriate control method is selected, and the control system is designed.

\section{Control system hardware design}

As for construction equipment space cross line automatic welding, due to welding of saddle trajectory to achieve the control object, data computation and so on system requirements, we must first consider the system operation speed; in addition to the work environment is bad, the on-site construction progress control strictly, equipment maintenance more difficult, but also consider the reliability and durability

control mode selection. Because the control precision of jacket automatic welding requirements is relatively high, the control system is more complicated, according to the control characteristics of jacket automatic welding machine, the idea of distributed, open and modular control system. For the control of multi DOF robot computer, centralized control and distributed control in two ways, the jacket of automatic welding machine including the input motion coordination control between the 
control and the input motion itself, require large amounts of computational resources, with a single controller to finish obviously unrealistic, therefore, the control system must be coordinated control the system structure of distributed multiple autonomous controller based on.

control system logical structure design. According to the jacket design idea of automatic welding machine control system and automatic welding machine control jacket convenience and computation time of strategy implementation and other issues, the logical structure of jacket automatic welding machine control system as shown in figure 4.

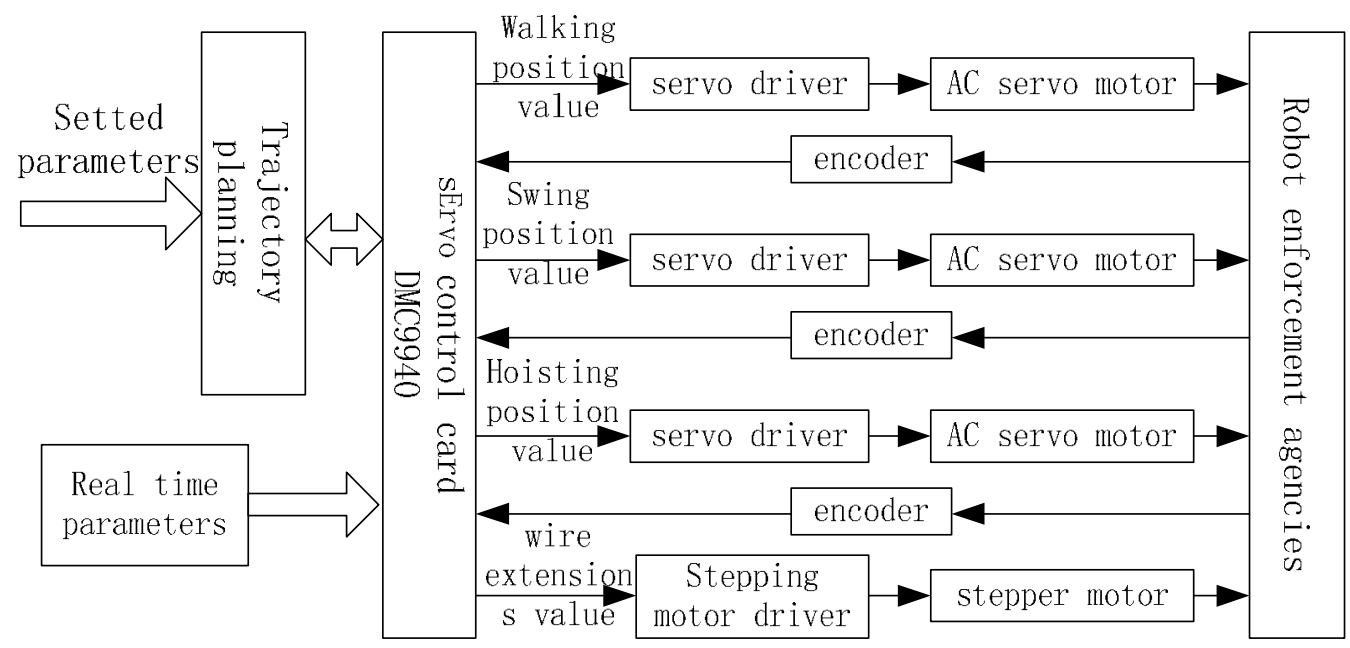

Fig4. Logical structure of control system

In the system, the DMC9940 GALIL motion control card is adopted, and the control of the motion system is realized by using the advanced language programming on the computer. Motor controller output interface board driver directly transmits a signal to the driving component, the driving control voltage generating motor components according to the input signal and subdivision parameter settings, the drive motor rotation, the high speed low torque motor is converted to automatic welding joints need low speed, increase the load capacity of motor

control system hardware structure design. After the input parameters and welding parameters, the welding machine control system first through the operation to get the desired trajectory, and then through the trajectory planning, the desired trajectory is converted to the generalized position coordinates of the drive joint. The robot control system through the encoder feedback signal detection, and compared with a given target position, according to the error between the continuous control, the actual position of the welding joint movement to the desired position. Trajectory planning and control in the upper computer by the software to achieve, control output by the motion control card and the drive is completed, and ultimately by the motor execution. The hardware structure of the control system is shown in figure 5 .

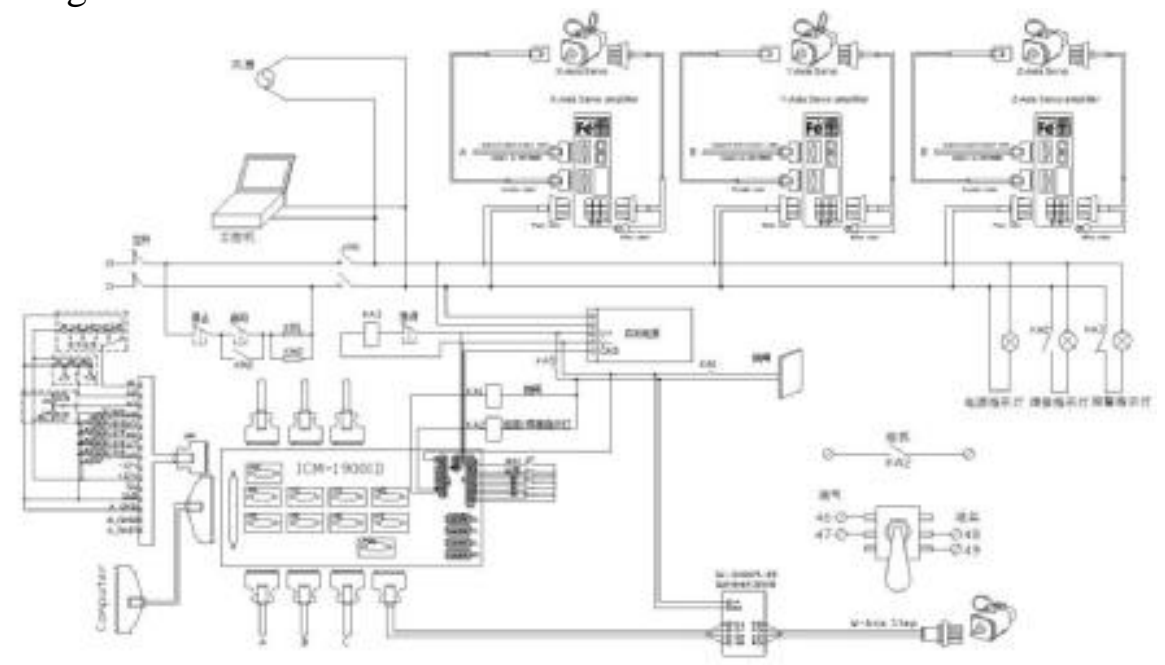


Fig5. Hardware schematic diagram of control system

In order to determine the motion status of the joint of the automatic welding machine in real time, the output shaft of the drive motor of each branch of the automatic welding machine is provided with an encoder. The output signal of the encoder is connected to the input terminal of the encoder motion control card interface board, the input signal can be read through the real-time encoder interface function in programming, timely access to the actual position of joint movement in the process of automatic welding machine.

\section{Control system software design}

Design of distributed control system The control system of automatic welding machine control system is based on the Visual Studio Microsoft 2008.Net framework, the program code is managed, stable and reliable. The jacket automatic welding machine adopts adaptive programming.

principle, control system can realize the input operation parameters, welding parameters, and the reasonable system configuration of existing file system parameters of the loading operation. The software has two display modes, namely state parameter display mode and status display mode, the jacket automatic welding control system also supports interrupt operation when welding, welding fault can be interrupted at any time during the welding process, and the interruption position teaching recording and playback. The simulation and setting of various possible situations are carried out according to the actual characteristics of welding.

Control process design. In view of the above control function and the need of the overall design idea, the software flow chart of the automatic welding machine control system is designed, as shown in figure 6. The first generation of welding trajectory using the trajectory planning, and then the control strategy is designed to make the welding torch tracking trajectory, the complex seam tracking problem into motor servo control problem.
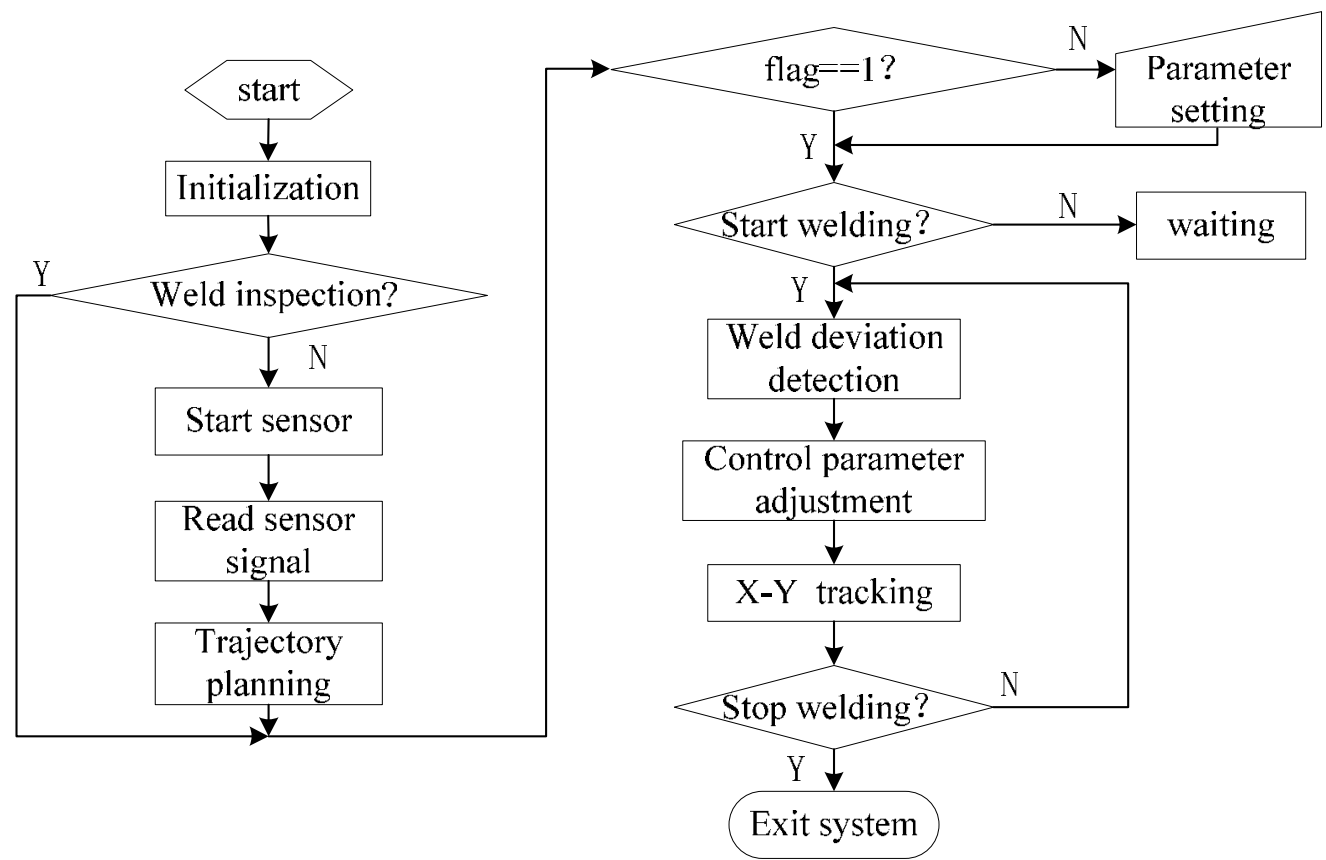

Fig6. Software design flow chart of control system

In the actual welding process, strong electromagnetic interference and high current caused the trajectory tracking error, which leads to inaccurate tracking or gun uncontrolled phenomenon. Design of variable parameter control system for large electromagnetic interference, use a parameter to change, can weaken the sudden increase of the step type interference signal, and can completely filter out the interference signal of certain frequency; on the other hand, motion control parameters in all position 
welding in the system is able to more accurately and quickly adapt to the random change of load and speed.

\section{Conclusions}

In this paper, the jacket space intersecting line distributed control technology based on spatial cross line trajectory analysis, establishes the mathematical model of the welding trajectory, realizes automatic multi axis motion control system.

It is verified that the control system can realize the precise control of the automatic welding movement mechanism of the pipe frame space intersection line, and the control precision error is less than $1 \%$, which meets the requirements of the actual welding control system.

At the same time, the technology can be used for the automatic welding of K type and Y type node of the jacket.

\section{References}

[1] S Weikerta, W Knapp. R-test, a new device for accuracy measurements on five axis machine tools [J]. Annals of the CIRP 2004, 53:429-432.

[2] V E Gough, S. G. Whitehall. Universal type test machine [C]. Proc.9th Int. Tech. Congress FISITA, 2002: 117-137.

[3] Zheng Aqi. Visual C++ tutorial, Second Edition. Beijing: Tsinghua University press,.2003.8 (In Chinese).

[4] Chen Pengchao, Yan Zheng and. PAW2000 all position automatic welding machine research paper. Anthology of pipeline Science (1999-2003), petroleum industry press, 2003. (In Chinese)

[5] Han Zandong et al. Parameter control system for pipe full position welding. Welding Journal, 2003; 24 (5): 1-3,9 (In Chinese)

[6] Jiang Shuying. Automatic control system of pipeline all position welding machine based on PMAC. Electric welding machine, 2002; 32 (9):25-27 (In Chinese)

[7] Information on http://www.skycontrol.net/flight-simulators/cae-awarded-contract-to-build-aw13 9-helicopter-simulator-for-agustawestland/ 